\title{
ON THE REGULARITY OF THE ANOSOV SPLITTING FOR TWISTED GEODESIC FLOWS
}

\author{
Gabriel P. Paternain
}

\begin{abstract}
Let $M$ denote a closed Riemannian manifold whose geodesic flow is Anosov. Given a real number $\lambda$ and a smooth one form $\theta$, consider the twisted geodesic flow obtained by twisting the canonical symplectic structure by the lift of $\lambda d \theta$ to the tangent bundle of $M$. For $\lambda$ in a certain open interval around the origin the twisted flow remains Anosov. We show that the Anosov splitting of the twisted geodesic flow is never of class $C^{1}$ unless $\lambda=0$.
\end{abstract}

\section{Introduction}

Let $M^{n}$ be a closed $n$-dimensional manifold endowed with a $C^{\infty}$ Riemannian metric $\langle$,$\rangle , and let \pi: T M \rightarrow M$ denote the canonical projection. Let $\omega_{0}$ denote the symplectic form on $T M$ obtained by pulling back the canonical symplectic form of $T^{*} M$ via the Riemannian metric. Let $H: T M \rightarrow \mathbb{R}$ be defined by

$$
H(x, v)=\frac{1}{2}\langle v, v\rangle .
$$

The Hamiltonian flow of $H$ with respect to $\omega_{0}$ gives rise to the geodesic flow of $M$. Let $\Omega$ be a closed 2-form of $M$ which does not vanish identically and consider the new symplectic form $\omega_{\lambda}$ defined as:

$$
\omega_{\lambda} \stackrel{\text { def }}{=} \omega_{0}+\lambda \pi^{*} \Omega, \quad \lambda \in \mathbb{R} .
$$

Such a form is called a twisted symplectic structure [1] and the Hamiltonian flow of $H$ with respect to $\omega_{\lambda}$ gives rise to a flow $\phi_{t}^{\lambda}: T M \rightarrow T M$ that we shall call twisted geodesic flow. This flow models the motion of a particle of unit mass and charge $\lambda$ under the effect of a magnetic field, whose Lorentz force $Y: T M \rightarrow T M$ is the bundle map uniquely determined by:

$$
\Omega_{x}(u, v)=\left\langle Y_{x}(u), v\right\rangle,
$$

for all $u$ and $v$ in $T_{x} M$ and all $x \in M$. Observe that $\phi_{t}^{\lambda}$ preserves all the energy levels $H=$ const, in particular $S M \stackrel{\text { def }}{=} H^{-1}(1 / 2)$. From now on let us consider the restriction of $\phi_{t}^{\lambda}$ to $S M$.

Various properties of these flows were studied in [22, 23]. For example, we showed that if we start with an Anosov geodesic flow $\phi_{t}^{0}$ and we increase the value of $\lambda$ we must exit the set of Anosov flows for some critical value $\lambda_{c}<\infty$

Received April 2, 1997.

Partially supported by grants from CSIC and CONICYT \# 301. 
and that the topological entropy presents a strict global maximum at $\lambda=0$ when restricted to $\left(-\lambda_{c}, \lambda_{c}\right)$.

In the present paper we shall study a new feature of the twisted geodesic flows, namely the regularity of the Anosov splitting. If $\lambda \in\left(-\lambda_{c}, \lambda_{c}\right)$, let us denote by $E_{\lambda}^{0} \oplus E_{\lambda}^{s} \oplus E_{\lambda}^{u}$ the Anosov splitting of $\phi_{t}^{\lambda}$, where $E_{\lambda}^{0}$ denotes the one dimensional subbundle associated with the flow direction and $E_{\lambda}^{s, u}$ denote the strong stable and strong unstable bundles respectively.

If $\operatorname{dim} M=2$ then $E_{\lambda}^{0} \oplus E_{\lambda}^{s}$ and $E_{\lambda}^{0} \oplus E_{\lambda}^{u}$ are both of class $C^{1, x} \log x$ by results of S.Hurder and A. Katok [16]. In particular, when $\lambda=0$, i.e. for geodesic flows, this implies that $E_{0}^{s}$ and $E_{0}^{u}$ are both of class $C^{1, x} \log x$ since the geodesic flow is of contact type. Also, if $M$ has 1/4-pinched negative sectional curvature, $E_{0}^{s}$ and $E_{0}^{u}$ are both of class $C^{1}[15]$. If one assumes that $E_{0}^{s}$ and $E_{0}^{u}$ are both of class $C^{\infty}$ then combining results of Y. Benoist, P. Foulon and F. Labourie [2] with results of G. Besson, G. Courtois and S. Gallot [3] it follows that $M$ must be locally symmetric, thus generalizing and improving previous results of M. Kanai, A. Katok and R. Feres [17, 7, 8, 9]. Most likely the same result is true assuming only that $E_{0}^{s}$ and $E_{0}^{u}$ are both of class $C^{2}$ but this is only known for surfaces [12] and for small deformations of hyperbolic metrics by results of $U$. Hamenstädt [13] and L. Flaminio [10]. We refer to [14] for more on the regularity of the Anosov splitting.

Our aim is to show that for twisted geodesic flows assuming $C^{1}$ regularity already implies rigidity provided that $\Omega$ is an exact form:

Theorem. Let $M$ be a closed Riemannian manifold whose geodesic flow is Anosov. Suppose $\Omega$ is exact. Then $E_{\lambda}^{s}$ and $E_{\lambda}^{u}$ are never both of class $C^{1}$ unless $\lambda=0$.

If the cohomology class of $\Omega$ is not trivial, the theorem is no longer true as it can be easily seen by looking at the case of a surface of constant negative curvature and $\Omega$ the area form. More generally consider a compact locally symmetric space of non-constant negative curvature $(n \geq 4)$. Let $J_{1}, \ldots, J_{d-1}$ be the parallel orthogonal endomorphisms defining the complex $(d=2)$, quaternionic $(d=4)$ or Cayley $(d=8)$ hyperbolic structure of $M$. If we consider the 2 -form $\Omega$ naturally associated each $J_{i}(1 \leq i \leq d-1)$ then it is straightforward to check that the splitting is $C^{\infty}$.

Problem. Are these are the only cases in which the splitting can be $C^{1}$ for $\lambda \neq 0$ ?

Observe that for surfaces, $E_{\lambda}^{s}$ and $E_{\lambda}^{u}$ are both of class $C^{1}$ if and only if $E_{\lambda}^{s} \oplus E_{\lambda}^{u}$ is of class $C^{1}$. If $\tau_{\lambda}$ denotes the one-form that vanishes on $E_{\lambda}^{s} \oplus E_{\lambda}^{u}$ and takes the value one on the vector field associated with $\phi_{t}^{\lambda}$, then the theorem is saying, for the surface case, that $\tau_{\lambda}$ is of class $C^{1}$ if and only if $\lambda=0$. Note that $\tau_{0}$ is $C^{\infty}$ and coincides with the contact form $\alpha$ of the geodesic flow.

J.F. Plante [25] gave the first examples of volume preserving Anosov flows for which the strong stable and unstable bundles are not both of class $C^{1}$. His 
examples are also volume preserving perturbations of Anosov geodesic flows, but he used the fact that the asymptotic cycle (cf. [26]) of the measure induced by the volume form was not zero for the perturbed flows. We shall see in Section 2 that $\phi_{t}^{\lambda}$ preserves the volume form $\alpha \wedge(d \alpha)^{n-1}$ and therefore the Liouville measure $\mu_{l}$ of $S M$. We shall show also in Section 2 that the asymptotic cycle of $\phi_{t}^{\lambda}$ with respect to $\mu_{l}$ vanishes for all $\lambda$ (provided that $M$ is not a 2 -torus). It follows that no argument like in [25] can be used to show the non-smoothness of the bundles $E_{\lambda}^{s}$ and $E_{\lambda}^{u}$, even in the surface case.

The proof of theorem will be based on a combination of a result of $U$. Hamenstädt in [13] and the theory of convex superlinear Lagrangians developed by John Mather and Ricardo Mañé (cf. [4, 19, 20, 21]). By writing $\Omega=d \theta$, the twisted geodesic flows can also be obtained as the Euler-Lagrange flows of the one-parameter family of Lagrangians

$$
L_{\lambda}(x, v)=\frac{1}{2}\langle v, v\rangle-\lambda \theta_{x}(v) .
$$

The energy of these Lagrangians is $E(x, v)=\frac{1}{2}\langle v, v\rangle$. As we shall explain in Section 3 , to each $L_{\lambda}$ one can attach the strict critical value $c_{0}\left(L_{\lambda}\right)$ as introduced by Mañé in [19]. The proof of the theorem splits into the three cases:

- $1 / 2>c_{0}\left(L_{\lambda}\right)$;

- $1 / 2=c_{0}\left(L_{\lambda}\right)$;

- $1 / 2<c_{0}\left(L_{\lambda}\right)$.

The three cases may indeed occur, as it is shown in [24], as long as we do not make any smoothness assumption on the bundles $E_{\lambda}^{s}$ and $E_{\lambda}^{u}$. Each case will give rise to different variational properties that will allow us to prove the theorem. We shall see that if $E_{\lambda}^{s}$ and $E_{\lambda}^{u}$ are both of class $C^{1}$ then the last two cases cannot occur and that in the first case we must have $\lambda=0$.

It is well known that for flows, a "choice of time" or equivalently, a choice of speed at which orbits travel gets reflected on the regularity of the corresponding strong stable and strong unstable distributions. The results of Hurder and Katok mentioned before imply that for a contact Anosov flow on a closed three manifold the Anosov splitting is $C^{1, x} \log x$. Our theorem is therefore saying that for $\operatorname{dim} M=2$ a twisted geodesic flow obtained from twisting an Anosov geodesic flow by an exact form $\Omega$ is never of contact type. As the referee pointed out this is rather surprising since it shows that in some sense the "best time" to describe the dynamics of the motion of a free particle under the effect of a magnetic field it is not the one associated to the Riemannian length. This observation naturally raises the following question posed by the referee: when can we change the speed of the flow to make it contact? The main result in [5] implies that if $1 / 2>c_{0}\left(L_{\lambda}\right)$ then the twisted geodesic flow is the reparametrization of a Finsler geodesic flow, which are known to be of contact type. However as we shall see in Section 4 after the proof of the theorem, our methods show, at least for surfaces, that if $1 / 2 \leq c_{0}\left(L_{\lambda}\right)$ then the twisted geodesic flow can never be reparametrized to make it of contact type. Examples with $1 / 2 \leq c_{0}\left(L_{\lambda}\right)$ do indeed exist (even 
for surfaces) as it was shown in [24]. The obstruction of generalizing this result to higher dimensions lies on the fact that Hamenstädt's methods in [13] (cf. Theorem 5.1 in the Appendix) definitely need a $C^{1}$ splitting. I do not know if this regularity assumption can be dropped.

Acknowledgement: I am grateful to the Institut Fourier, Grenoble, for hospitality while this work was completed and to the referee for several comments and suggestions.

\section{Preliminaries}

We describe in this section some of the basic geometry of the twisted geodesic flows.

Let us begin by fixing on $M$ a smooth Riemannian metric $\langle$,$\rangle . Let \pi$ : $T M \rightarrow M$ denote the canonical projection and let $K: T T M \rightarrow T M$ denote the connection map. The latter is defined giving its value on each fibre setting

$$
K_{v}(\xi)=\left.\frac{D Z}{d t}\right|_{t=0},
$$

where $Z:(-\epsilon, \epsilon) \rightarrow T M$ verifies $Z(0)=v, Z^{\prime}(0)=\xi$, and $\frac{D}{d t}$ denotes covariant derivative along $\pi \circ Z$.

It is well known that $T T M$ splits as the direct sum of the vertical and the horizontal subbundles. The vertical fibre on $v$ is given by

$$
V(v)=\operatorname{Ker} d_{v} \pi,
$$

and the horizontal fibre on $v$ is defined as

$$
H(v)=\operatorname{Ker} K_{v} .
$$

Thus $T_{v} T M$ can be identified with $T_{\pi(v)} M \oplus T_{\pi(v)} M$, and hence we write in the sequel

$$
\xi=\left(\xi_{1}, \xi_{2}\right)
$$

where $\xi_{1}=d_{v} \pi(\xi)$ and $\xi_{2}=K_{v}(\xi)$ for every $\xi$ in $T_{v} T M$. The symplectic structure $\omega_{0}$ described in the Introduction can be written as

$$
\omega_{0}(\xi, \eta)=\langle d \pi(\xi), K(\eta)\rangle-\langle K(\xi), d \pi(\eta)\rangle .
$$

The contact form $\alpha$ can be written as

$$
\alpha_{v}(\xi)=\langle d \pi(\xi), v\rangle
$$

and we have that $\omega_{0}=-d \alpha$.

Fix a closed two-form $\Omega$ in $M$ such that $\Omega$ does not vanish identically. For any $\lambda \in \mathbb{R}$ define $\omega_{\lambda}$ by

$$
\omega_{\lambda}=\omega_{0}+\lambda \pi^{*} \Omega
$$


Consider the Hamiltonian $H: T M \rightarrow \mathbb{R}$ given by $H(v)=\frac{1}{2}\langle v, v\rangle$. Let $Y: T M \rightarrow T M$ be the bundle map such that

$$
\Omega_{x}(u, v)=\left\langle Y_{x}(u), v\right\rangle
$$

for all $u$ and $v$ in $T_{x} M$ and all $x$ in $M$. Denote by

$$
X_{\lambda}: T M \rightarrow T T M
$$

the symplectic gradient of $H$ with respect to $\omega_{\lambda}$. Since the identity

$$
d_{v} H(\xi)=\omega_{0}\left(X_{\lambda}(v), \xi\right)+\lambda\left\langle Y\left(d_{v} \pi\left(X_{\lambda}(v)\right)\right), d_{v} \pi(\xi)\right\rangle
$$

holds for every $\xi$ in $T_{v} T M$, the identity

$$
\left\langle\xi_{2}, v\right\rangle=\left\langle X_{\lambda}^{1}(v), \xi_{2}\right\rangle-\left\langle X_{\lambda}^{2}(v), \xi_{1}\right\rangle+\lambda\left\langle\left(X_{\lambda}^{1}(v)\right), \xi_{1}\right\rangle
$$

is valid for all $\xi_{1}$ and $\xi_{2} \in T_{\pi(v)} M$ (obviously we made use of the identification $\xi=\left(\xi_{1}, \xi_{2}\right)$ as it was explained before and $\left(X_{\lambda}^{1}, X_{\lambda}^{2}\right)$ are the horizontal and vertical components of $\left.X_{\lambda}\right)$. Therefore

$$
X_{\lambda}(v)=(v, \lambda Y(v))
$$

for every $v$ in $T M$. It is easily seen from this equation that a curve is an integral curve of $X_{\lambda}$ if and only if it is of the form $t \mapsto(\gamma(t), \dot{\gamma}(t)) \in T M$ and satisfies the equation

$$
\frac{D}{d t} \dot{\gamma}=\lambda Y(\dot{\gamma})
$$

which is nothing but Newton's law of motion.

Suppose now that $\phi_{t}^{0}: S M \rightarrow S M$ is an Anosov flow and let $\left(-\lambda_{c}, \lambda_{c}\right)$ denote the maximal interval such that for $\lambda \in\left(-\lambda_{c}, \lambda_{c}\right), \phi_{t}^{\lambda}$ is an Anosov flow.

Lemma 2.1. Take $\lambda \in\left(-\lambda_{c}, \lambda_{c}\right)$. There is no periodic orbit of $\phi_{t}^{\lambda}$ whose projection to $M$ is null-homotopic. If $\sigma$ denotes a non-trivial free homotopy class of $M$, then there exists a unique closed orbit of $\phi_{t}^{\lambda}$ such that its projection to $M$ belongs to the homotopy class $\sigma$.

Proof. Recall that if $\phi_{t}^{0}$ is Anosov, there exists a unique closed geodesic in the class $\sigma$ and there is no closed geodesic homotopic to zero. The lemma follows right away from the fact that $\phi_{t}^{\lambda}$ is topologically equivalent to $\phi_{t}^{0}$ by a homeomorphism isotopic to the identity. 
Let us prove now that $\alpha \wedge(d \alpha)^{n-1}$ is invariant under $\phi_{t}^{\lambda}$ acting on $S M$. It suffices to show that

$$
d\left(i_{X_{\lambda}}\left(\alpha \wedge(d \alpha)^{n-1}\right)\right)=0 .
$$

But this last equality is a consequence the following lemma

\section{Lemma 2.2.}

$$
i_{X_{\lambda}}\left(\alpha \wedge(d \alpha)^{n-1}\right)=\left(-\omega_{\lambda}\right)^{n-1}
$$

Proof. By the definition of $\omega_{\lambda}$

$$
(d \alpha)^{n-1}=\left(-\omega_{\lambda}+\lambda \pi^{*} \Omega\right)^{n-1} .
$$

Note that on $S M$ we have

$$
\alpha\left(X_{\lambda}\right)=1
$$

The lemma follows now from observing that

$$
\alpha \wedge \omega_{\lambda}^{i} \wedge\left(\pi^{*} \Omega\right)^{n-i-1}=0,
$$

unless $i=n-1$.

Let $\mu_{l}$ denote the probability measure on $S M$ induced by the volume form $\alpha \wedge(d \alpha)^{n-1}$. For any $\lambda$, the twisted geodesic flow leaves $\mu_{l}$ invariant.

Let us show that the asymptotic cycle of $\mu_{l}$ vanishes provided that $M$ is not diffeomorphic to a 2-torus. If $\varphi$ denotes any closed one-form on $S M$ of class $C^{1}$, we need to show that

$$
\int_{S M} \varphi\left(X_{\lambda}\right) d \mu_{l}=0
$$

But using Lemma 2.2 we have

$$
\int_{S M} \varphi\left(X_{\lambda}\right) \alpha \wedge(d \alpha)^{n-1}=\int_{S M} i_{X_{\lambda}}\left(\alpha \wedge(d \alpha)^{n-1}\right) \wedge \varphi=(-1)^{n} \int_{S M}\left(\omega_{\lambda}\right)^{n-1} \wedge \varphi .
$$

If $n \geq 3$, the form $\left(\omega_{\lambda}\right)^{n-1}$ is always exact. If $n=2$ and $M$ is not diffeomorphic to a 2 -torus, the form $\omega_{\lambda}$ is still exact because $\pi^{*} \Omega$ is also exact, as can be easily seen using the (non-zero) Euler class of the bundle $\pi: S M \rightarrow M$. It follows that if $M$ is not diffeomorphic to a 2-torus the form $\left(\omega_{\lambda}\right)^{n-1}$ is always exact. If we write $\left(\omega_{\lambda}\right)^{n-1}=d \beta_{\lambda}$ we have

$$
\int_{S M}\left(\omega_{\lambda}\right)^{n-1} \wedge \varphi=\int_{S M} d\left(\beta_{\lambda} \wedge \varphi\right)=0
$$

and thus the asymptotic cycle of $\mu_{l}$ vanishes for all $\lambda$. 


\section{Critical values of Lagrangians}

We shall describe in this section the main results that we need from the theory of convex superlinear Lagrangians as developed by Mather and Mañé (cf. $[4,19,20,21])$.

Let $M^{n}$ be a closed manifold and let $L: T M \rightarrow \mathbb{R}$ be a $C^{\infty}$ Lagrangian satisfying the following hypothesis:

- Convexity. For all $x \in M$, the restriction of $L$ to $T_{x} M$ has everywhere positive definite Hessian.

- Superlinear growth. Let || || denote a Riemannian metric on $M$. Then

$$
\lim _{\|v\| \rightarrow \infty} \frac{L(x, v)}{\|v\|}=+\infty
$$

uniformly on $x \in M$. This condition is clearly independent of the choice of Riemannian metric, since $M$ is compact.

The Euler-Lagrange equation,

$$
\frac{d}{d t}\left(\frac{\partial L}{\partial v}(x, \dot{x})\right)-\frac{\partial L}{\partial x}(x, \dot{x})=0
$$

generates a smooth complete flow $\phi_{t}: T M \rightarrow T M$ which is defined as follows. Given $(x, v) \in T M$, consider the unique solution $x: \mathbb{R} \rightarrow M$ of the EulerLagrange equation with initial conditions

$$
x(0)=x, \quad \dot{x}(0)=v .
$$

Now define $\phi_{t}: T M \rightarrow T M$ by

$$
\phi_{t}(x, v)=(x(t), \dot{x}(t)) .
$$

Recall that the energy $E: T M \rightarrow \mathbb{R}$ is defined by

$$
E(x, v)=\frac{\partial L}{\partial v}(x, v) \cdot v-L(x, v) .
$$

Since $L$ is autonomous, $E$ is a first integral of the flow $\phi_{t}$.

Recall that the action of the Lagrangian $L$ on an absolutely continuous curve $u:[a, b] \rightarrow M$ is defined by

$$
A_{L}(u)=\int_{a}^{b} L(u(t), \dot{u}(t)) d t .
$$

Given two points, $x_{1}$ and $x_{2}$ in $M$ and $T>0$ denote by $\mathcal{C}_{T}\left(x_{1}, x_{2}\right)$ the set of absolutely continuous curves $u:[0, T] \rightarrow M$, with $u(0)=x_{1}$ and $u(T)=x_{2}$. For each $k \in \mathbb{R}$ we define the action potential $\Phi_{k}: M \times M \rightarrow \mathbb{R}$ by

$$
\Phi_{k}\left(x_{1}, x_{2}\right)=\inf \left\{A_{L+k}(u): u \in \cup_{T>0} \mathcal{C}_{T}\left(x_{1}, x_{2}\right)\right\} .
$$

Mañé showed $[19,4]$ that there exists $c(L) \in \mathbb{R}$ such that

- if $k<c(L)$, then $\Phi_{k}\left(x_{1}, x_{2}\right)=-\infty$, for all $x_{1}$ and $x_{2}$; 
- if $k \geq c(L)$, then $\Phi_{k}\left(x_{1}, x_{2}\right)>-\infty$ for all $x_{1}$ and $x_{2}$ and $\Phi_{k}$ is a Lipschitz function;

- if $k \geq c(L)$, then

$$
\Phi_{k}\left(x_{1}, x_{3}\right) \leq \Phi_{k}\left(x_{1}, x_{2}\right)+\Phi_{k}\left(x_{2}, x_{3}\right),
$$

for all $x_{1}, x_{2}$ and $x_{3}$ and

$$
\Phi_{k}\left(x_{1}, x_{2}\right)+\Phi_{k}\left(x_{2}, x_{1}\right) \geq 0,
$$

for all $x_{1}$ and $x_{2}$;

- if $k>c(L)$, then for $x_{1} \neq x_{2}$ we have

$$
\Phi_{k}\left(x_{1}, x_{2}\right)+\Phi_{k}\left(x_{2}, x_{1}\right)>0 .
$$

Observe that in general the action potential $\Phi_{k}$ is not symmetric, however defin$\operatorname{ing} d_{k}: M \times M \rightarrow \mathbb{R}$ by

$$
d_{k}\left(x_{1}, x_{2}\right)=\Phi_{k}\left(x_{1}, x_{2}\right)+\Phi_{k}\left(x_{2}, x_{1}\right),
$$

the properties above say that $d_{k}$ is a metric for $k>c(L)$ and a pseudometric for $k=c(L)$. The number $c(L)$ is called the critical value of $L$.

It is important for our purposes to indicate that the results above also hold for coverings of $M$, i.e. suppose $\widehat{M}$ is a covering of $M$ with covering projection $p$. Take the lift of the Lagrangian $L$ to $\widehat{M}$ which is given by

$$
\widehat{L}(\widehat{x}, \widehat{v})=L(p(\widehat{x}), d p(\widehat{v})) .
$$

Then we define for each $k \in \mathbb{R}$ the action potential $\widehat{\Phi}_{k}$ just as above and the results hold for $\widehat{L}$. Thus we have a critical value for $\widehat{L}$. Let $\bar{M}$ denote the abelian covering of $M$ which is defined as the covering of $M$ whose fundamental group is the kernel of the Hurewicz homomorphism $\pi_{1}(M) \mapsto H_{1}(M, \mathbb{R})$. The abelian covering of $M$ gives rise to the critical value

$$
c_{a}(L) \stackrel{\text { def }}{=} c(\bar{L}) \text {. }
$$

We shall need the following result $[19,4]$.

Theorem 3.1. Let $\sigma$ denote a non-trivial free homotopy class of $M$ and suppose that $k>c(L)$. Then, there exists a periodic orbit in the energy level $k$ such that its projection to $M$ belongs to $\sigma$ and that minimizes the action $A_{L+k}$ over the set of absolutely continuous closed curves in the class $\sigma$. Conversely, if there exists an absolutely continuous closed curve $u$ in the class $\sigma$ that minimizes the action $A_{L+k}$ among the set of all curves in the class $\sigma$, then $u$ gives rise to a periodic orbit of the Euler-Lagrange flow with energy $k$. 
Proof. This theorem is not explicitly stated in [19, 4], but its proof follows immediately from the proofs of Theorem X and Corollaries 4.3 and 4.4 in [4]. For the reader's convenience we shall explain the main ideas in its proof. For each $T>0$, let $\mathcal{C}_{T}$ be the space of all absolutely continuous closed curves $u$ : $[0, T] \rightarrow M$ in the class $\sigma$. Let $a$ denote the infimum of $A_{L+k}$ over the space $\cup_{T>0} \mathcal{C}_{T}$. Since $k>c(L)$ this infimum is $>-\infty$. Consider for each $T>0$ a Tonelli minimizer $\gamma_{T}$ (cf. [18, p. 46] and [21]) of the action $A_{L}$ over $\mathcal{C}_{T}$. Take a sequence $\gamma_{T_{n}}$ such that $A_{L+k}\left(\gamma_{T_{n}}\right)$ approaches $a$. The main point here is that $\left\{T_{n}\right\}$ has a convergent subsequence that approaches a number $0<T_{0}<\infty$. This is because $k>c(L)$ and $\sigma$ is a non-trivial homotopy class. Using this fact one shows that the Tonelli minimizers $\gamma_{T_{n}}$ have a subsequence that converges to a closed curve of period $T_{0}$ that is a periodic solution of the Euler-Lagrange equation. Using that this curve minimizes $A_{L+k}$ over the space $\cup_{T>0} \mathcal{C}_{T}$ one shows that its energy is precisely $k$. For the converse statement in the theorem, observe that if $u:\left[0, T_{0}\right] \rightarrow M$ minimizes $A_{L+k}$ over the space $\cup_{T>0} \mathcal{C}_{T}$ it must be also a Tonelli minimizer and therefore a periodic solution with energy $k$.

Let us recall now the main concepts introduced by Mather in [21].

Let $\mathcal{M}(L)$ be the set of probabilities on the Borel $\sigma$-algebra of $T M$ that have compact support and are invariant under the flow $\phi_{t}$. Let $H_{1}(M, \mathbb{R})$ be the first real homology group of $M$. Given a closed one-form $\omega$ on $M$ and $\rho \in H_{1}(M, \mathbb{R})$, let $\langle\omega, \rho\rangle$ denote the integral of $\omega$ on any closed curve in the homology class $\rho$. If $\mu \in \mathcal{M}(L)$, its homology is defined as the unique $\rho(\mu) \in H_{1}(M, \mathbb{R})$ such that

$$
<\omega, \rho(\mu)>=\int \omega d \mu
$$

for all closed one-forms on $M$. The integral on the right-hand side is with respect to $\mu$ with $\omega$ considered as a function $\omega: T M \rightarrow \mathbb{R}$. The function $\rho: \mathcal{M}(L) \rightarrow H_{1}(M, \mathbb{R})$ is surjective [21].

The action of $\mu \in \mathcal{M}(L)$ is defined by

$$
A_{L}(\mu)=\int L d \mu
$$

Finally we define the function $\beta: H_{1}(M, \mathbb{R}) \rightarrow \mathbb{R}$ by

$$
\beta(\gamma)=\inf \left\{A_{L}(\mu): \rho(\mu)=\gamma\right\}
$$

The function $\beta$ is convex and superlinear and the infimum can be shown to be a minimum [21] and the measures at which the minimum is attained are called minimizing measures. In other words, $\mu \in \mathcal{M}(L)$ is a minimizing measure iff

$$
\beta(\rho(\mu))=A_{L}(\mu)
$$

Mañé $[19,4]$ established a connection between the critical values of a Lagrangian and the convex dual of Mather's $\beta$ function. He showed that 


$$
c(L)=-\min \left\{\int L d \mu: \mu \in \mathcal{M}(L)\right\} .
$$

Let us recall how the convex dual $\alpha: H^{1}(M, \mathbb{R}) \rightarrow \mathbb{R}$ of $\beta$ is defined. Since $\beta$ is convex and superlinear we can set

$$
\alpha([\omega])=\max \left\{<\omega, \gamma>-\beta(\gamma): \gamma \in H_{1}(M, \mathbb{R})\right\},
$$

where $\omega$ is any closed one-form whose cohomology class is [ $\omega$ ]. The function $\alpha$ is also convex and superlinear. Mather [21] showed that

$$
\alpha([\omega])=-\min \left\{\int(L-\omega) d \mu: \mu \in \mathcal{M}(L)\right\}
$$

and therefore using (3) we obtain the remarkable equality

$$
c(L-\omega)=\alpha([\omega]),
$$

for any closed one-form $\omega$ whose cohomology class is $[\omega]$. Finally, Mañé defined the strict critical value of $L$ as

$$
c_{0}(L) \stackrel{\text { def }}{=} \min \left\{c(L-\omega):[\omega] \in H^{1}(M, \mathbb{R})\right\}=-\beta(0) .
$$

We showed in [24] that the strict critical value of $L$ equals the critical value of the lift of $L$ to the abelian covering of $M$, that is, $c_{a}(L)=c_{0}(L)$.

\section{Proof of the theorem}

Since $\Omega$ is exact we can write $\Omega=d \theta$ for some smooth one-form $\theta$. As in the Introduction, let us define

$$
L_{\lambda}(x, v)=\frac{1}{2}\langle v, v\rangle-\lambda \theta_{x}(v) .
$$

The energy function associated with $L_{\lambda}$ is

$$
E(x, v)=\frac{1}{2}\langle v, v\rangle .
$$

Let $\mathcal{L}_{\lambda}: T M \rightarrow T^{*} M$ denote the Legendre transform associated with $L_{\lambda}$ and let $\omega_{\text {can }}$ denote the canonical symplectic form of $T^{*} M$. It is well known that the Euler-Lagrange flow of $L_{\lambda}$ can be obtained as the Hamiltonian flow of $E$ with respect to the symplectic form $\mathcal{L}_{\lambda}^{*} \omega_{\text {can }}$. An easy computation shows that

$$
\mathcal{L}_{\lambda}^{*} \omega_{\text {can }}=\omega_{0}+\lambda \pi^{*} d \theta
$$

and thus the Euler-Lagrange flow of $L_{\lambda}$ coincides with the twisted geodesic flow.

As in the Introduction let $\tau_{\lambda}$ denote the one-form that vanishes on $E_{\lambda}^{s} \oplus E_{\lambda}^{u}$ and takes the value one on the vector field $X_{\lambda}$. If the splitting is of class $C^{1}$ then $\tau_{\lambda}$ is also of class $C^{1}$ and $d \tau_{\lambda}$ is a continuous two-form invariant under the twisted geodesic flow. U. Hamenstädt showed in [13], for the geodesic flow 
case, that any continuous invariant exact two-form must be a constant multiple of the symplectic form provided that the splitting is of class $C^{1}$. Hamenstädt's proof carries over to the case of twisted geodesic flows without major changes, provided that $\Omega$ is an exact form. However, for completeness sake we include a sketch of the proof of this fact in the appendix. It follows that there exists a constant $c$ such that:

$$
d \tau_{\lambda}=c \omega_{\lambda}
$$

and thus

$$
d\left(\tau_{\lambda}+c \alpha-c \lambda \pi^{*} \theta\right)=0
$$

Let us write

$$
\varphi_{\lambda} \stackrel{\text { def }}{=} \tau_{\lambda}+c \alpha-c \lambda \pi^{*} \theta .
$$

Then $\varphi_{\lambda}$ is a smooth closed one-form. Using (2) we obtain

$$
\varphi_{\lambda}\left(X_{\lambda}\right)(v)=1+c-c \lambda \theta_{\pi v}(v) .
$$

Integrating the last equality with respect to $\mu_{l}$ and using that the asymptotic cycle of $\mu_{l}$ vanishes we have

$$
0=1+c-c \lambda \int_{S M} \theta_{\pi v}(v) d \mu_{l}
$$

On the other hand $\mu_{l}$ is invariant under the flip $v \mapsto-v$ and since $\theta_{\pi v}(v)=$ $-\theta_{\pi v}(-v)$, the integral of $\theta_{\pi v}(v)$ over $S M$ must vanish and thus $c=-1$. Replacing in (5) we finally obtain

$$
\varphi_{\lambda}\left(X_{\lambda}\right)(v)=\lambda \theta_{\pi v}(v) .
$$

It is well known that the map $\pi^{*}: H^{1}(M, \mathbb{R}) \rightarrow H^{1}(S M, \mathbb{R})$ is an isomorphism (provided that $M$ is not diffeomorphic to a 2-torus). Therefore there exist a closed smooth one-form $\delta$ in $M$ and a smooth function $f: S M \rightarrow \mathbb{R}$ such that

$$
\varphi_{\lambda}=\pi^{*} \delta+d f
$$

and hence equation (6) gives

$$
\delta_{\pi v}(v)+d f\left(X_{\lambda}\right)(v)=\lambda \theta_{\pi v}(v) .
$$

The proof splits now into three cases.

- $1 / 2>c_{0}\left(L_{\lambda}\right)$.

By equality (4) we can take a closed one-form $\omega$ such that $c_{0}\left(L_{\lambda}\right)=c\left(L_{\lambda}-\omega\right)$. Let $\gamma:[0, T] \rightarrow M$ denote a periodic solution of the Euler-Lagrange equation of $L_{\lambda}$ with energy 1/2. By Lemma 2.1 the homotopy class $\sigma$ of $\gamma$ is non-trivial and $\gamma$ is the only periodic solution in $\sigma$ with energy $1 / 2$. Let $\eta:[0, R] \rightarrow M$ denote the unique periodic solution in the homotopy class $-\sigma$. Since the homotopy 
class of $-\gamma$ is $-\sigma$ and the homotopy class of $-\eta$ is $\sigma$ it follows from Theorem 3.1 that

$$
\begin{aligned}
& A_{L_{\lambda}-\omega+1 / 2}(\eta) \leq A_{L_{\lambda}-\omega+1 / 2}(-\gamma) \\
& A_{L_{\lambda}-\omega+1 / 2}(\gamma) \leq A_{L_{\lambda}-\omega+1 / 2}(-\eta)
\end{aligned}
$$

But

$$
\begin{gathered}
A_{L_{\lambda}-\omega+1 / 2}(\eta)=R-\int_{\eta} \omega-\lambda \int_{\eta} \theta \\
A_{L_{\lambda}-\omega+1 / 2}(-\gamma)=T+\int_{\gamma} \omega+\lambda \int_{\gamma} \theta \\
A_{L_{\lambda}-\omega+1 / 2}(\gamma)=T-\int_{\gamma} \omega-\lambda \int_{\gamma} \theta \\
A_{L_{\lambda}-\omega+1 / 2}(-\eta)=R+\int_{\eta} \omega+\lambda \int_{\eta} \theta
\end{gathered}
$$

therefore

$$
\begin{aligned}
& R \leq T+\int_{\eta+\gamma} \omega+\lambda \int_{\eta+\gamma} \theta, \\
& T \leq R+\int_{\eta+\gamma} \omega+\lambda \int_{\eta+\gamma} \theta .
\end{aligned}
$$

Since the cycle $\eta+\gamma$ is homologous to zero and $\eta$ and $\gamma$ are solutions of the Euler-Lagrange equation we have using (7) that

$$
\lambda \int_{\eta+\gamma} \theta=0
$$

Also, since $\omega$ is closed

$$
\int_{\eta+\gamma} \omega=0
$$

It follows from (8) and (9) that $T=R$ and thus

$$
A_{L_{\lambda}-\omega+1 / 2}(\eta)=A_{L_{\lambda}-\omega+1 / 2}(-\gamma),
$$

and by Theorem 3.1, $-\gamma$ must be a solution of the Euler-Lagrange equation since $\eta$ is a minimizer in the class $-\sigma$; in fact it follows that $\eta=-\gamma$. Therefore $t \mapsto \gamma(-t)$ and $t \mapsto \gamma(t)$ are both solutions of equation (1) and thus

$$
\lambda Y(\dot{\gamma}(-t))=-\lambda Y(\dot{\gamma}(-t)),
$$

which implies that $\lambda Y(\dot{\gamma}(0))=0$. Since the periodic orbits are dense and $Y$ does not vanish identically we must have $\lambda=0$ thus concluding the proof of the theorem in the case $1 / 2>c_{0}\left(L_{\lambda}\right)$.

- $1 / 2=c_{0}\left(L_{\lambda}\right)$. 
Take a minimizing measure $\mu$ such that $\rho(\mu)=0$. It satisfies

$$
\beta(0)=\int L_{\lambda} d \mu \text {. }
$$

A result of M.J. Dias Carneiro [6] assures that the support of $\mu$ is contained in the energy level $-\beta(0)=c_{0}\left(L_{\lambda}\right)=1 / 2$ therefore

$$
-1 / 2=1 / 2-\lambda \int_{S M} \theta_{\pi v}(v) d \mu .
$$

Integrating both sides of (7) with respect to $\mu$ and using the fact that $\mu$ is invariant we obtain

$$
\int_{S M} \delta_{\pi v}(v) d \mu=\lambda \int_{S M} \theta_{\pi v}(v) d \mu
$$

Therefore

$$
<\delta, \rho(\mu)>=1
$$

Since $\rho(\mu)=0$, we obtain a contradiction and hence the case $1 / 2=c_{0}\left(L_{\lambda}\right)$ cannot occur if the splitting is of class $C^{1}$.

- $1 / 2<c_{0}\left(L_{\lambda}\right)$.

We shall need the following proposition which has independent interest.

Proposition 4.1. Suppose that there exists $\lambda$ such that for all $\lambda^{\prime}$ with $\lambda^{\prime} \leq|\lambda|$, the energy level $1 / 2$ of $L_{\lambda^{\prime}}$ is Anosov. Then, if $1 / 2<c_{0}\left(L_{\lambda}\right)$, there exists a periodic solution $x(t)$ of the Euler-Lagrange equation of $L_{\lambda}$ with energy $1 / 2$ such that if $T$ denotes a period, then $\left.x\right|_{[0, T]}$ is homologous to zero and

$$
A_{L_{\lambda}+1 / 2}\left(\left.x\right|_{[0, T]}\right)<0 .
$$

Proof. Since $c_{a}\left(L_{\lambda}\right)=c_{0}\left(L_{\lambda}\right)$ it follows that if $1 / 2<c_{0}\left(L_{\lambda}\right)$, there exist $T_{0}>0$ and an absolutely continuous closed curve $u:\left[0, T_{0}\right] \rightarrow M$ homologous to zero such that

$$
A_{L_{\lambda}+1 / 2}(u)<0 .
$$

Let us denote by $\sigma$ the free homotopy class of $u$. For any $\tau \geq T_{0}$, let $\mathcal{C}_{\tau}(\sigma)$ denote the set of all absolutely continuous closed curves $w:[0, t] \rightarrow M$ such that $t \leq \tau$ and such that the free homotopy class of $w$ is $\sigma$. The same arguments that prove Tonelli's Theorem (cf. [18, p. 46] and [21]) allow us to conclude that the action $A_{L_{\lambda}+1 / 2}$ takes a finite minimum value on the set $\mathcal{C}_{\tau}(\sigma)$ and minimizers are periodic solutions of the Euler-Lagrange equation of $L_{\lambda}$. In other words, for each $\tau$ there exists a solution $x: \mathbb{R} \rightarrow M$ of the Euler-Lagrange equation and $T \in[0, \tau]$ such that $\left.x\right|_{[0, T]}$ minimizes the action $A_{L_{\lambda}+1 / 2}$ on the set $\mathcal{C}_{\tau}(\sigma), x$ is periodic with period $T$ and the homotopy class of $\left.x\right|_{[0, T]}$ is $\sigma$. By (10) the 
minimum value has to be negative and therefore $T \neq 0$. Let $k$ be the energy of the solution $x$.

Let us define for each $s>0$ the following function:

$$
F(s) \stackrel{\text { def }}{=} \int_{0}^{s T}\left(L_{\lambda}+1 / 2\right)\left(x_{s}, \dot{x}_{s}\right) d t
$$

where $x_{s}(t):[0, s T] \rightarrow M$ is defined as $x_{s}(t)=x\left(\frac{t}{s}\right)$. Then

$$
F(s)=\left(\frac{k}{s}+\frac{s}{2}\right) T-\lambda \int_{x_{s}} \theta .
$$

Let us compute $F^{\prime}(1)$. Since $\int_{x_{s}} \theta$ does not depend on $s$ we have,

$$
F^{\prime}(s)=\left(\frac{-k}{s^{2}}+\frac{1}{2}\right) T
$$

and thus

$$
F^{\prime}(1)=\left(\frac{1}{2}-k\right) T
$$

Now observe that since $\left.x\right|_{[0, T]}$ minimizes the action $A_{L_{\lambda}+1 / 2}$ on the set $\mathcal{C}_{\tau}(\sigma)$ we must have $F^{\prime}(1) \leq 0$ and therefore $k \geq 1 / 2$. In fact if $T<\tau$ then $F^{\prime}(1)=0$ and therefore $k=1 / 2$.

Observe that there exists $k_{0}$ such that for all $(x, v)$ with energy $\geq k_{0}$ we have $L_{\lambda}(x, v) \geq 0$. Therefore since $A_{L_{\lambda}+1 / 2}\left(\left.x\right|_{[0, T]}\right)<0$ it follows that $k<k_{0}$.

Let $g: T M \rightarrow T M$ be the map $(x, v) \mapsto(x, \sqrt{2 k} v)$. An easy computation shows that

$$
g^{*} \omega_{\lambda}=\sqrt{2 k}\left(\omega_{0}+\frac{\lambda}{\sqrt{2 k}} \pi^{*} d \theta\right)
$$

and that $g$ sends $S M$ into the energy level $k$. It follows that

$$
g^{*}\left(\left.X_{\lambda}\right|_{\text {level } k}\right)=\left.\sqrt{2 k} X_{\frac{\lambda}{\sqrt{2 k}}}\right|_{S M} .
$$

Since $k \geq 1 / 2$ we have that $\frac{\lambda}{\sqrt{2 k}} \leq|\lambda|$. Using the fact that for all $\lambda^{\prime} \leq|\lambda|$, the energy level $1 / 2$ of $L_{\lambda^{\prime}}$ is Anosov and Lemma 2.1 we deduce that there exists a unique periodic orbit of the Euler-Lagrange flow of $L_{\lambda}$ with energy $k$ such that its projection to $M$ belongs to the class $\sigma$. Let us denote by $T_{k}$ the period of this orbit. The map $k \mapsto T_{k}$ is smooth and therefore is bounded on $\left[1 / 2, k_{0}\right]$. It follows that for some $\tau$ sufficiently large, the minimizer $\left.x\right|_{[0, T]}$ has period $T$ strictly less than $\tau$ and therefore it has energy precisely $1 / 2$. Since $\left.x\right|_{[0, T]}$ is homotopic to a curve homologous to zero, it must be itself homologous to zero and finally since it is a minimizer we must have

$$
A_{L_{\lambda}+1 / 2}\left(\left.x\right|_{[0, T]}\right)<0,
$$

as desired. 
Proposition 4.1 implies that there exists a periodic solution $x(t)$ of the EulerLagrange equation with energy $1 / 2$ such that if $T$ denotes a period, then $\gamma \stackrel{\text { def }}{=}$ $\left.x\right|_{[0, T]}$ is homologous to zero and

$$
A_{L_{\lambda}+1 / 2}(\gamma)<0
$$

Equivalently

$$
T-\lambda \int_{\gamma} \theta<0
$$

which implies that

$$
\lambda \int_{\gamma} \theta>0
$$

However, since $\gamma$ is homologous to zero, equation (7) implies that

$$
\lambda \int_{\gamma} \theta=0
$$

This contradiction shows that the case $1 / 2<c_{0}\left(L_{\lambda}\right)$ cannot occur if the splitting is of class $C^{1}$ thus concluding the proof of the theorem.

We shall explain now why our methods also show the following result.

Proposition 4.2. If there exists a time change that makes the flow $\phi_{t}^{\lambda}$ of contact type, then $1 / 2>c_{0}\left(L_{\lambda}\right)$, provided that $M$ is a surface.

Proof. Suppose that the exists a smooth positive function $g: S M \rightarrow \mathbb{R}$ and a smooth one form $\Theta$ such that $\Theta\left(g X_{\lambda}\right)=1$ and such that the flow of $g X_{\lambda}$ leaves $\Theta$ invariant. Cartan's formula implies right away that $i_{g X_{\lambda}} d \Theta=0$. Since $g$ is non-zero, we obtain $i_{X_{\lambda}} d \Theta=0$ and therefore $d \Theta$ is invariant under $\phi_{t}^{\lambda}$. Since $\operatorname{dim} M=2$, ergodicity of $\phi_{t}^{\lambda}$ implies that $d \Theta$ must be a constant multiple of the symplectic form. Therefore there exists a constant $c$ such that

$$
d \Theta=c \omega_{\lambda},
$$

and thus $\Theta+c \alpha-c \lambda \pi^{*} \theta$ is a closed one form that we denote as in the proof of the theorem by $\varphi_{\lambda}$. Observe that

$$
\varphi_{\lambda}\left(X_{\lambda}\right)(v)=\frac{1}{g}+c-c \lambda \theta_{\pi v}(v) .
$$

Integrating the last equality with respect to $\mu_{l}$ and using that the asymptotic cycle of $\mu_{l}$ vanishes we have

$$
0=\int_{S M} \frac{1}{g} d \mu_{l}+c-c \lambda \int_{S M} \theta_{\pi v}(v) d \mu_{l} .
$$

On the other hand $\mu_{l}$ is invariant under the flip $v \mapsto-v$ and since $\theta_{\pi v}(v)=$ $-\theta_{\pi v}(-v)$, the integral of $\theta_{\pi v}(v)$ over $S M$ must vanish and thus $c=-\int_{S M} \frac{1}{g} d \mu_{l}<0$. Suppose now that $\mu$ is any measure whose support is 
contained in $S M$, invariant under $\phi_{t}^{\lambda}$ and with $\rho(\mu)=0$. Integrating the last equality with respect to $\mu$ we obtain (just as in the proof of the theorem):

$$
0=\int_{S M} \frac{1}{g} d \mu+c A_{L_{\lambda+1 / 2}}(\mu)
$$

Since $g$ is a positive function and $c$ is negative we must have

$$
A_{L_{\lambda+1 / 2}}(\mu)>0 \text {. }
$$

Now the proof of the theorem shows that this can only happen if $1 / 2>c_{0}\left(L_{\lambda}\right)$.

The difficulty of generalizing this argument to higher dimensions is that one would need a result like Theorem 5.1 in the Appendix but dropping the hypothesis that the splitting is $C^{1}$.

\section{Appendix}

In this appendix we sketch a proof of the following result:

Theorem 5.1. Let $\eta$ be a continuous exact 2-form in $S M$ invariant under the twisted geodesic flow $\phi_{t}^{\lambda}$ induced by the closed 2 -form $\Omega$. Suppose that $\Omega$ is exact and the Anosov splitting of $\phi_{t}^{\lambda}$ is of class $C^{1}$. Then $\eta$ is a constant multiple of $\omega_{\lambda}$.

Proof. This theorem was proved by U. Hamenstädt in [13] for geodesic flows. We shall explain now why her proof extends to the case of twisted geodesic flows provided that $\Omega$ is an exact form. Observe that the theorem is a straightforward consequence of ergodicity if $n=2$.

Let us write $\eta=d \tau$ and $\nu \stackrel{\text { def }}{=}-\alpha+\lambda \pi^{*} \theta$, where $\theta$ is a 1-form such that $d \theta=\Omega$. Clearly $d \nu=\omega_{\lambda}$. First note that since $\eta$ is $\phi_{t}^{\lambda}$-invariant, $i_{X_{\lambda}} \eta=0$. Also there exists a bundle map $L: E_{\lambda}^{s} \oplus E^{u} \rightarrow E_{\lambda}^{s} \oplus E_{\lambda}^{u}$ such that $L$ is $\phi_{t}^{\lambda}$-invariant and

$$
\eta(x, y)=\omega_{\lambda}(L x, y)
$$

for $x$ and $y$ in $E_{\lambda}^{s} \oplus E_{\lambda}^{u}$.

Let us define

$$
A=\int_{S M} \nu \wedge\left(\omega_{\lambda}\right)^{n-1}
$$

Note that $A \neq 0$ since by Stokes theorem, A also equals the integral of the volume form $\left(\omega_{\lambda}\right)^{n}$ on the unit disk bundle in $T M$.

Consider the function $F: \mathbb{R} \rightarrow \mathbb{R}$ given by

$$
F(r)=\int_{S M}(\tau-r \nu) \wedge\left(\omega_{\lambda}\right)^{n-1}
$$


Since $F^{\prime}(r)=-A$, it follows that there exists $c \in \mathbb{R}$ such that $F(c)=0$. Let us set $\beta=\tau-c \nu$. Clearly $d \beta$ is $\phi_{t}^{\lambda}$-invariant and $i_{X_{\lambda}} d \beta=0$. By ergodicity, there exist constants $c_{i}$ such that

$$
d \beta^{i} \wedge\left(\omega_{\lambda}\right)^{n-1-i}=c_{i}\left(\omega_{\lambda}\right)^{n-1},
$$

and thus

$$
\nu \wedge d \beta^{i} \wedge\left(\omega_{\lambda}\right)^{n-1-i}=c_{i} \nu \wedge\left(\omega_{\lambda}\right)^{n-1} .
$$

Integrating by parts one finds that

$$
c_{i-1} F(c)=c_{i} A,
$$

and therefore all the $c_{i}$ must vanish. It follows that $L$ can be written as $c I d+B$, where $B: E_{\lambda}^{s} \oplus E_{\lambda}^{u} \rightarrow E_{\lambda}^{s} \oplus E_{\lambda}^{u}$ is a nilpotent map. Next we note that $E_{\lambda}^{s}$ and $E_{\lambda}^{u}$ are invariant subspaces for $B$. Let $B^{s}$ and $B^{u}$ denote the map induced by $B$ on $E_{\lambda}^{s}$ and $E_{\lambda}^{u}$ respectively. We shall show that $B^{s}$ and $B^{u}$ vanish. Let $Q(v) \stackrel{\text { def }}{=} \operatorname{Ker} B^{s}(v)$. Choose an open dense $\phi_{t}^{\lambda}$-invariant set $U \subset S M$ on which $Q$ is a continuous subbundle of $E_{\lambda}^{s}$. Now the key step is Lemma 4.3 in [13] which shows that $\left.Q\right|_{U}$ is an integrable subbundle. (here one uses that the splitting is $C^{1}$ ). Using the holonomy transport along the weak unstable foliation (one also needs here the splitting to be $C^{1}$ ) we can construct as in [13, Lemma 3.4] a $C^{0}$-foliation of $S \tilde{M}$, the unit sphere bundle of the universal covering of $M$. Since $\phi_{t}^{\lambda}$ is topologically conjugate to the geodesic flow $\phi_{t}^{0}$ it can be easily seen that the space of leaves $\mathcal{F}$ of the lift of the weak unstable foliation to $S \tilde{M}$ is topologically also a sphere $S^{n-1}$. Moreover the $C^{0}$-foliation descends to the space of leaves $\mathcal{F}$ and induces a $C^{0}$-foliation, which by construction, is invariant under the induced action of $\pi_{1}(M)$ on $\mathcal{F}$. The latter is in turn conjugate to the corresponding action of $\pi_{1}(M)$ on the sphere at infinity of the geodesic flow. It follows now from a result of P. Foulon [11] that such a foliation must be trivial and therefore $B^{s}$ must vanish identically. The argument to prove the vanishing of $B^{u}$ is completely similar.

\section{References}

1. V. I. Arnold and A. B. Givental, Symplectic geometry, Dynamical Systems IV, Encyclopedia of Mathematical Sciences, Springer Verlag, Berlin, 1990.

2. Y. Benoist, P. Foulon, and F. Labourie, Flots d'Anosov a distributions stable et inestable différentiables, Jour. Amer. Math. Soc. 5 (1992), 33-74.

3. G. Besson, G. Courtois, and S. Gallot, Entropies et rigidités des espaces localement symétriques de courbure strictement négative, Geom. Funct. Anal. 5 (1995), 731-799.

4. G. Contreras, J. Delgado, and R. Iturriaga, Lagrangian flows: The dynamics of globally minimizing orbits II, Bull. Brazilian Math. Soc. 28 (1997), 155-196.

5. G. Contreras, R. Iturriaga, G. P. Paternain, and M. Paternain, Lagrangian graphs, minimizing measures and Mañé's critical values, preprint 1997, available via internet at "http://www.ma.utexas.edu/mp_arc".

6. M. J. Dias Carneiro, On minimizing measures of the action of autonomous Lagrangians, Nonlinearity 8 (1995), 1077-1085. 
7. R. Feres, A. Katok, Invariant tensor fields of dynamical systems with pinched Liapunov exponents and rigidity of geodesic flows, Ergodic Theory Dynamical Systems 9 (1989), 427-432.

8. _ Anosov flows with smooth foliations and rigidity of geodesic flows in threedimensional manifolds of negative curvature, Ergodic Theory Dynamical Systems 10 (1990), 657-670.

9. R. Feres, Geodesic flows on manifolds with negative with smooth horospheric foliations, Ergodic Theory Dynamical Systems 11 (1991), 653-686.

10. L. Flaminio, Local entropy rigidity for hyperbolic manifolds, Comm. Anal. Geom. 3 (1995), 555-596.

11. P. Foulon, Feuilletages des sphéres et dynamiques Nord-Sud, C. R. Acad. Sci Paris t. 318 (1994), 1041-1042.

12. E. Ghys, Flots d'Anosov dont les feuilletages stables sont différentiables, Ann. Sci. École Norm. Sup. 20 (1987), 251-270.

13. U. Hamenstädt, Invariant two-forms for geodesic flows, Math. Ann. 301 (1995), 677-698.

14. B. Hasselblatt, Regularity of the Anosov splitting and of horospherical foliations, Ergodic Theory Dynamical Systems 14 (1994), 645-666.

15. M. Hirsch and C. Pugh, Smoothness of horocycle foliations, J. Diff. Geom. 10 (1975), $225-238$.

16. S. Hurder and A. Katok, Differentiability, rigidity and Godbillon-Vey classes for Anosov flows, Inst. Hautes Etudes Sci. Publ. Math. 72 (1990), 5-61

17. M. Kanai, Geodesic flows on negatively curved manifolds with smooth stable and unstable foliations, Ergodic Theory Dynamical Systems 8 (1988), 215-240.

18. R. Mañé, Global variational methods in conservative dynamics, 18 Colóquio Brasileiro de Matemática, IMPA, 1991.

19. R. Mañé, Lagrangian flows: the dynamics of globally minimizing orbits, International Conference on Dynamical Systems (Montevideo, 1995), 120-131, Pitman Res. Notes Math. Ser., 362, Longman, Harlow, 1996.

20. R. Mañé, Generic properties and problems of minimizing measures of Lagrangian systems, Nonlinearity 9 (1996), 273-310.

21. J. Mather, Action minimizing measures for positive definite Lagrangian systems, Math. Z. 207 (1991), 169-207.

22. G. P. Paternain and M. Paternain, Anosov geodesic flows and twisted symplectic structures, International Congress on Dynamical Systems in Montevideo (a tribute to Ricardo Mañé), F. Ledrappier, J. Lewowicz, S. Newhouse eds, Pitman Research Notes in Math. 362 (1996), 132-145.

23. First derivative of topological entropy for Anosov geodesic flows in the presence of magnetic fields, Nonlinearity 10 (1997), 121-131.

24. _ Critical values of autonomous Lagrangian systems, Comment. Math. Helvetici $\mathbf{7 2}$ (1997), 481-499.

25. J. F. Plante, Anosov flows, Amer. J. Math. 94 (1972), 729-754

26. R. Schwartzman, Asymptotic cycles, Ann. of Math. (2) 66 (1957), 270-284.

Centro de Matemática, Facultad de Ciencias, Eduardo Acevedo 1139, Montevideo CP 11200, URUGUAY

E-mail address: gabriel@cmat.edu.uy 